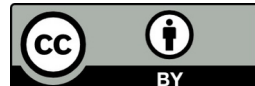

\title{
Estrategias didácticas y la comunicación, en los niños y niñas de 5 años de la Villa Monte Castillo: Catacaos, Piura, Perú
}

Didactic strategies and communication, in children and 5-year-old girls from Villa Monte Castillo: Catacaos, Piura, Peru

Estratégias didáticas e comunicação em meninos e meninas de 5 anos de Villa Monte Castillo: Catacaos, Piura, Peru

\section{ARTÍCULO ORIGINAL}

Jaimin Murillo Antón

https://orcid.org/0000-0002-8069-0640

jaiminmurillo@upeu.edu.pe

Universidad Peruana Unión

Lima - Perú
Salomón Vásquez Villanueva

https://orcid.org/0000-0001-8824-6176

salomonv@upeu.edu.pe

Universidad Peruana Unión

Lima - Perú

Recibido 07 de Abril 2021 | Arbitrado y aceptado 19 de Junio 2021 | Publicado en 03 Setiembre 2021

\section{RESUMEN}

Con el objeto determinar en qué medida se relacionan las estrategias didácticas (narrativa, poética, lectura, descriptiva, lúdicas, teatro, canto y dibujo) y la comunicación (familiar y escolar), en un estudio básico y correlación, de enfoque cuantitativo; el estudio fue realizado con la participación de 40 niños y niñas de educación inicial, en la edad de 5 años, en la Villa Monte Castillo, a quienes se les aplicó un cuestionario para medir las dos variables: estrategias didácticas y comunicación. Los resultados indican que no existe evidencia estadísticamente significativa ( $\mathrm{rs}=0.289, \mathrm{p}=0.071$ ), que muestre la existencia de relación entre la variable estrategias didácticas y comunicación. Solamente el canto tiene una relación significativa con la comunicación, el 0.827. En conclusión, no existe relación entre las variables estudiadas.

Palabras claves: estrategias didácticas y comunicación escolar

\section{ABSTRACT}

In order to determine to what extent didactic strategies (narrative, poetic, reading, descriptive, playful, theater, singing and drawing) and communication (family and school) are related, in a basic study and correlation, with a quantitative approach; The study was carried out with the participation of 40 boys and girls of initial education, at the age of 5, in Villa Monte Castillo, to whom a questionnaire was applied to measure the two variables: didactic strategies and communication. The results indicate that there is no statistically significant evidence ( $\mathrm{rs}=0.289, \mathrm{p}$ $=0.071$ ), which shows the existence of a relationship between the variable didactic strategies and communication. Only singing has a significant relationship with communication, 0.827 . In conclusion, there is no relationship between the variables studied.

Keywords: didactic strategies and school communication

\section{RESUMO}

Para determinar em que medida as estratégias didáticas (narrativa, poética, leitura, descritiva, lúdica, teatro, canto e desenho) e comunicação (família e escola) se relacionam, em um estudo básico e correlação, com uma abordagem quantitativa; $\mathrm{O}$ estudo foi realizado com a participação de 40 meninos e meninas do ensino inicial, aos 5 anos, em Villa Monte Castillo, aos quais foi aplicado um questionário para mensurar as duas variáveis: estratégias didáticas $\mathrm{e}$ comunicação. Os resultados indicam que não há evidências estatisticamente significativas (rs $=0,289, \mathrm{p}=0,071)$, o que mostra a existência de relação entre a variável estratégias de ensino e comunicação. Apenas o canto tem relação significativa com a comunicação, 0,827 Em conclusão, não há relação entre as variáveis estudadas.

Palavras-chave: estratégias de ensino e comunicação escolar. 


\section{INTRODUCCIÓN}

El estudio se realizó sobre dos variables: las estrategias didácticas (narrativa, poética, lectura, descriptiva, lúdicas, teatro, canto y dibujo) y la comunicación (familiar y escolar), buscando la posibilidad de mejorar las estrategias didácticas y la comunicación en la comunidad escolar.

Estrategias didácticas para niños de 5 años de edad

Melquiades (2014) considera que las estrategias didácticas para la enseñanza de los niños son: los juegos, cuya estrategia permite fundamentalmente el aprendizaje lógicomatemático, el cual permite el desarrollo cognitivo, mediante el "razonamiento, la imaginación, la creación y la experimentación”. Orellana-García y Melo-Hurtado (2014) hablan de alfabetización inicial, en el sentido de lectura y escritura, permitiendo la adquisición del lenguaje oral que las antecede, para quienes la metodología más eficientes es ELLCO (Early Language and Literacy Classroom Observation), propuesta por Smith, Brady \& Clark-Chiarelli, 2002.

Mendoza y Pabón (2013) realizan un estudio sobre el desarrollo del pensamiento lógicomatemático, en estudiantes pre escolares, cuya metodología propuesta comprende las nociones básicas: "clasificación, seriación y del concepto de identificación de atributos", estas nacen de la observación directa realizada sobre el proceso enseñanza aprendizaje, permitiendo al niño la construcción del pensamiento lógico.

Por su parte, Telpis y Terán (2014) consideran que la literatura infantil se ha constituido en una buena y excelente estrategia para el proceso enseñanza aprendizaje en la educación infantil, tal estrategia permite y facilita el desarrollo de las destrezas y del pensamiento lógico, en niños de 4 a 5 años de edad. También, para los mismos, permite ejercer la solución de problemas, la toma de decisiones, la creatividad, la imaginación, la intuición, la lógica, la educción social, entre otros.

Para Montenegro y Valencia (2013), los niños de 4 a 5 años aprenden, mejoran sus aprendizajes, sus habilidades, sus capacidades, mediante la elaboración de material didáctico, el cual sea dinámico y motivador, además de ser fácil en su realización. Los niños y las niñas aprenden haciendo, elaborando los materiales didácticos.

Comunicación familiar

Para Guaita (2010), la comunicación emocional de los padres es muy importante, cuya comunicación es expresada mediante las relaciones interpersonales, generando la interpretación de las intenciones, también de las emociones de los demás. En este mismo sentido, se destaca la importancia del desarrollo de la "adquisición de las competencias 
emocionales". Las relaciones familiares son valiosas, muy importantes, imprescindibles "para el desarrollo de las emociones, la capacidad para reconocerlas y expresarlas". De acuerdo con Valdés y Spencer (2011), en la familia no solamente es comunicación verbal, sino extra verbal. Se cultivan y desarrollan otros factores; por ejemplo, el factor socioeconómico, determinantes para el ejercicio de la comunicación familiar y del entorno en forma general, específicamente en niños de 4 a 5 años de edad, en cuya edad sobresale y se destaca el desarrollo psicomotor.

Garcés y Palacio (2010) destacan la importancia de la comunicación familiar, mucho más en los asentamientos humanos subnormales, en la cual destaca la presencia y participación directas de la madre, mucho más en las familias monoparentales. La comunicación de la madre es afectiva, además de reguladora, cuyos factores negativos que afectan y disminuyen la comunicación son: "la separación de los padres, el maltrato psicológico y físico, la crisis económica y el consumo de drogas".

Desarrollo del lenguaje

El desarrollo del lenguaje en los niños se constituye en un proceso, de acuerdo con Campo (2010), para quien el desarrollo físico corresponde a los procesos evolutivos del niño, particularmente durante los primeros años de vida; estos procesos se constituyen en la base del desarrollo psicológico y del mismo desarrollo del lenguaje. Pues el desarrollo físico y sicológico, ambos tienen de intermedio el desarrollo motor. En la perspectiva de Alvites (2012), el lenguaje es un hecho, un fenómeno lingüístico bastante complejo; también es concebido con la equivalencia de medio, herramienta comunicativa, cuyo desarrollo depende de los procesos sociales de las personas y de la sociedad en su conjunto, cuyo instrumento, además, permite la incorporación de los sujetos a la sociedad, razón por la cual el leguaje tiene un componente pragmático, el cual es desarrollado durante los primeros años de la existencia humana; es decir, en el ámbito de la familia, en el escenario denominado hogar.

Según Paredes (2011), el leguaje viene a ser un instrumento básico de comunicación, el cual debe ser estimulado desde los primeros años de vida, cuyo medio tiene relación con el conocimiento, con la conciencia, con las ideas, cuyo protagonista es el niño en su conexión con el mudo que lo rodea. En este sentido y en este contexto, tiene mucha significación la estimulación temprana para el cultivo y desarrollo del lenguaje en el hogar, especialmente durante la edad comprendida entre 4 y 5 años, evitando la presencia de problemas posteriores; por ejemplo, los problemas sociales, así como los problemas emocionales, observados durante los aprendizajes del niño. 
Para Trujillo (2013), los niños presentan o experimentan un desarrollo infantil totalmente complejo, cuyo proceso se da en un mundo de cambios constantes, de transformaciones, generadas por diversos aspectos, muy importantes para su desarrollo psíquico y lingüístico, sin dejar al margen su desarrollo físico en un universo de movimientos relacionados con su estructura corporal y motriz. Así, para la educación inicial, se debe proveer al niño los medios y los instrumentos más requeridos y necesarios, con el propósito de fortalecer su aprendizaje y desarrollo multidisciplinario.

\section{METODOLOGÍA}

Tipo de investigación

De acuerdo con Hernández Sampieri, Fernández Collado y Baptista Lucio (2014), el estudio es no experimental, corresponde al tipo descriptivo, correlacional y transversal. Es de tipo descriptivo, porque se describió estadísticamente la frecuencia, la intensidad, cómo se relacionan las variables, objeto de estudio: estrategias didácticas y comunicación. Es correlacional, se estudió la correlación de las variables referidas. Es transversal, porque se estudió las variables solamente durante un segmento temporal, el año 2014.

Diseño de la investigación

El presente trabajo tiene diseño de investigación no experimental, transeccional, por eso se

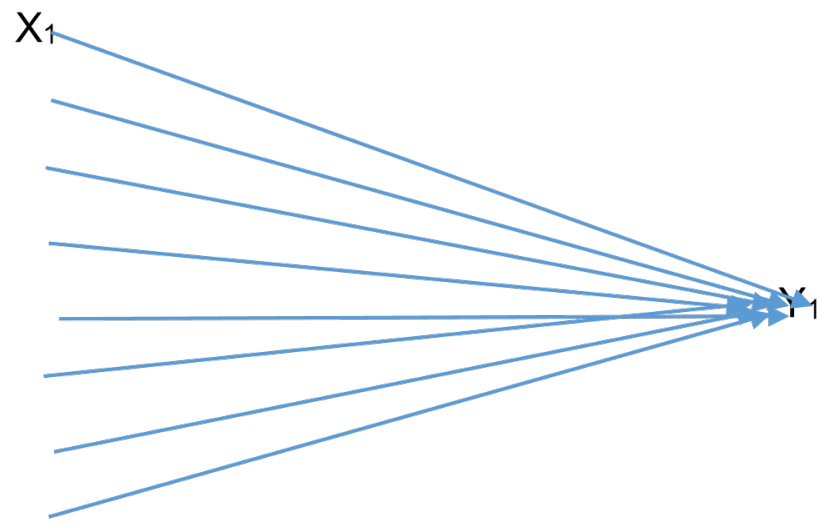

X 1

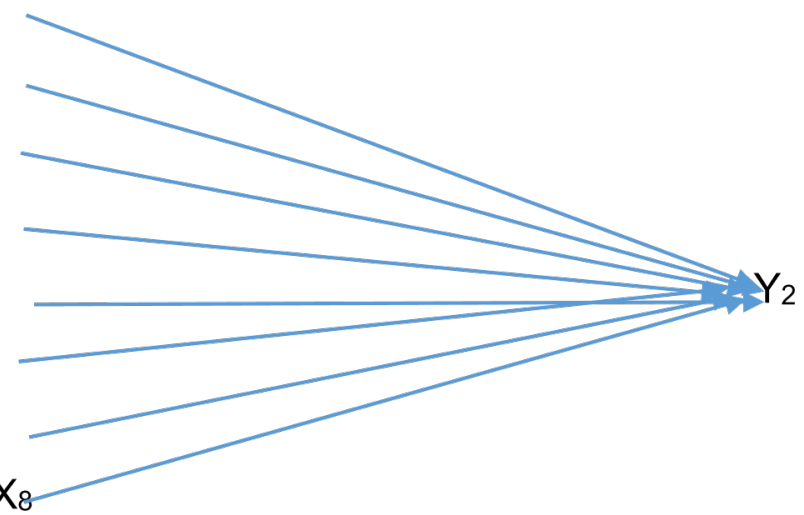
recabará datos en un solo momento, con el propósito de describir y determinar la correlación de las variables predictoras y criterio: estrategias didácticas $\mathrm{y}$ comunicación, incluyendo sus respectivas dimensiones, con los diseños siguientes: $\begin{array}{lllllll}\mathrm{X}_{2} & \mathrm{X}_{3} & \mathrm{X}_{4} & \mathrm{X}_{5} & \mathrm{X}_{6} & \mathrm{X}_{7} & \mathrm{X}\end{array}$

$\mathrm{X}_{2} \mathrm{X}_{3} \mathrm{X}_{4} \mathrm{X}_{5} \mathrm{X}_{6} \mathrm{X}_{7}$

Donde: 
$\mathrm{X} 1 \quad$ Estrategias narrativas,

X2 Estrategias poéticas

X3 Estrategias de lectura

X4 Estrategias descriptivas

X5 Estrategias lúdicas

X6 Estrategias de teatro

X7 Estrategias canto

X8 Estrategias de dibujo

X Estrategias didácticas

Y1 Comunicación familiar

Y2 Comunicación escolar

Y Comunicación

Participantes en la investigación

En la investigación participaron 40 estudiantes de la IE José Carlos Mariátegui La Chira, Piura, Catacaos. La participación fue determinada por conveniencia, sin recurrir a parámetros estadísticos, por ser un número bastante pequeño.

Técnicas de recolección de datos

Para la recolección de los datos sobre las estrategias didácticas y comunicación, se recurrió a la técnica de la encuesta, para lo cual se elaboró el respectivo instrumento, el cual fue validado mediante el juicio de expertos y los respectivos asesores de la tesis.

Técnicas para el procesamiento y análisis de datos obtenidos

Los datos organizados y presentados en tablas, luego del análisis de regresión múltiple, cuya fórmula para la correlación múltiple es:

$R=\frac{\sum_{i=0}^{n}(X i-X)(Y i-Y)}{\sqrt{\left[\left(\sum_{i=0}^{n}(X i-X)\right]\left[\left(\sum_{i=0}^{n}(Y i-Y)\right]\right.\right.}}$

Donde:

$\mathrm{N}=$ Número de sujetos

$\Sigma x y=$ Suma de la multiplicación de cada valor de $\mathrm{x}$ por cada valor de $\mathrm{y}$

$\Sigma \mathrm{x}=$ Suma de los valores de $\mathrm{x}$

$\Sigma \mathrm{y}=$ Suma de los valores de $\mathrm{y}$

$\Sigma \mathrm{x}^{2}$ y $\Sigma \mathrm{y}^{2}=$ Suma de cada uno de los valores de X y Y elevados al cuadrado. 
Descripción del instrumento utilizado

El instrumento utilizado fue un cuestionario denominado Estrategias didácticas y comunicación, elaborado por el investigador y con la opinión de los expertos. El instrumento está constituido por dos partes: "Información demográfica" e "Información sobre las variables de investigación: estrategias didácticas y comunicación”. La "Información demográfica" comprende cinco datos: género, edad, lugar donde estudio, lugar donde labora y año de promoción. Por su parte, la "Información sobre las variables de investigación: estrategias didácticas y comunicación”, comprende 30 ítems, distribuidos 23 para la variable: estrategias didácticas y 7 para la variable: comunicación. Los ítems de la variable: estrategias didácticas, fueron distribuidos en ocho dimensiones: Estrategias narrativas (1- 3), estrategias poéticas (4-6), estrategias de lectura (711), estrategias descriptivas (12-14), estrategias lúdicas (15-16), estrategias de teatro (17-19), estrategias de canto (20-21), estrategias de dibujo (22-23). Los ítems de la variable: comunicación, fueron distribuidos en dos dimensiones: comunicación familiar (24-27) y comunicación escolar $(28-$

$30)$.

Este instrumento fue revalidado por juicio de expertos en materia educativa, quienes validaron las preguntas que fueran las pertinentes para esta investigación; además se validó semántica y gramaticalmente. También fue sometido a la prueba de alfa de Crombach, para corroborar su revalidación, con un alfa de 0.95 de confiabilidad.

Tabla 2.

Resultados de validez del instrumento de investigación

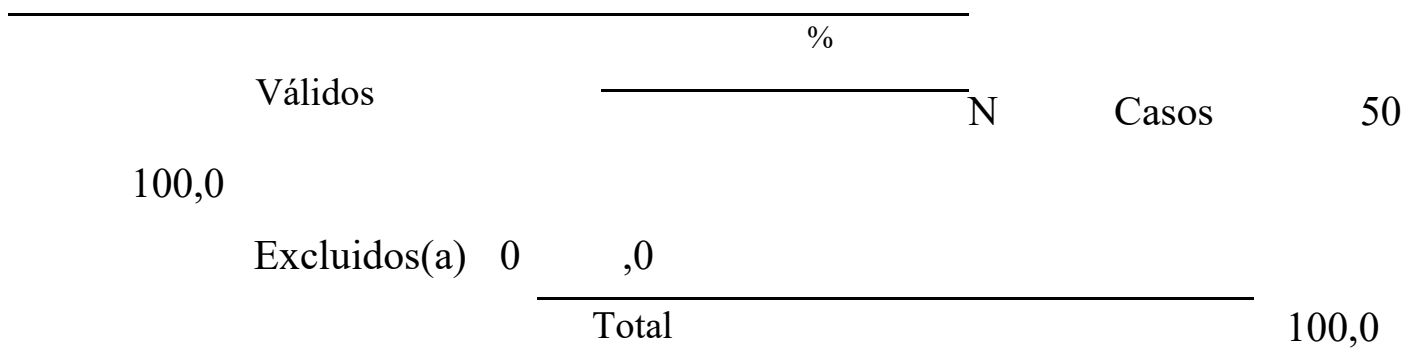

40

Eliminación por lista basada en todas las variables del procedimiento.

Tabla 3.

Estadísticos de fiabilidad 
El método que se utiliza para comprobar la fiabilidad del cuestionario es el Alfa de Cronbach. En este sentido, se trata de un índice de consistencia interna que tiene valores entre 0 y 1 , sirve para comprobar si el instrumento evaluado recopila información defectuosa y, por tanto, no nos llevaría a conclusiones equivocadas o si se trata de un instrumento fiable que hace mediciones estables y consistentes. Alfa es, en efecto, un coeficiente de correlación al cuadrado el cual, a grandes rasgos, mide la homogeneidad de las preguntas promediando todas las correlaciones entre los ítems para observar que efectivamente, se aproximan. La ecuación de Alfa de Corbacho es:

$$
\alpha=\frac{n p}{1+p(n-1)}
$$

Donde $\mathrm{n}=$ el número de ítems $\mathrm{p}=\mathrm{el}$ promedio de las correlaciones lineales entre cada uno de los ítems. La estimación de Alfa de Cronbach es alta, lo que garantiza que las puntuaciones percibidas son datos fiables de las respectivas características consideradas.

\section{RESULTADOS}

Relación entre estrategias didácticas y comunicación

Tabla 4

Relación entre la variable estrategias didácticas y comunicación en niños de 5 años

\begin{tabular}{llll}
\hline & \multicolumn{3}{c}{ Comunicación } \\
\cline { 3 - 4 } & $\mathrm{n}$ & $\mathrm{p}$ & $\mathrm{r}_{\mathrm{s}}$ \\
\hline Estrategias didácticas & 40 & 0.071 & 0.289
\end{tabular}

Al examinar la relación de estas variables: estrategias didácticas y comunicación, se determina que no existen evidencia estadísticamente significativa ( $\mathrm{rs}=0.289, \mathrm{p}=0.071$ ) que permitan la demostración de la existencia de relación significativa entre las referidas variables.

Relación entre estrategias didácticas y comunicación familiar

Tabla 5

Relación entre las dimensiones de las estrategias didácticas y comunicación familiar 
Comunicación familiar

Estrategias didácticas

$\mathrm{p} \quad \mathrm{r}$

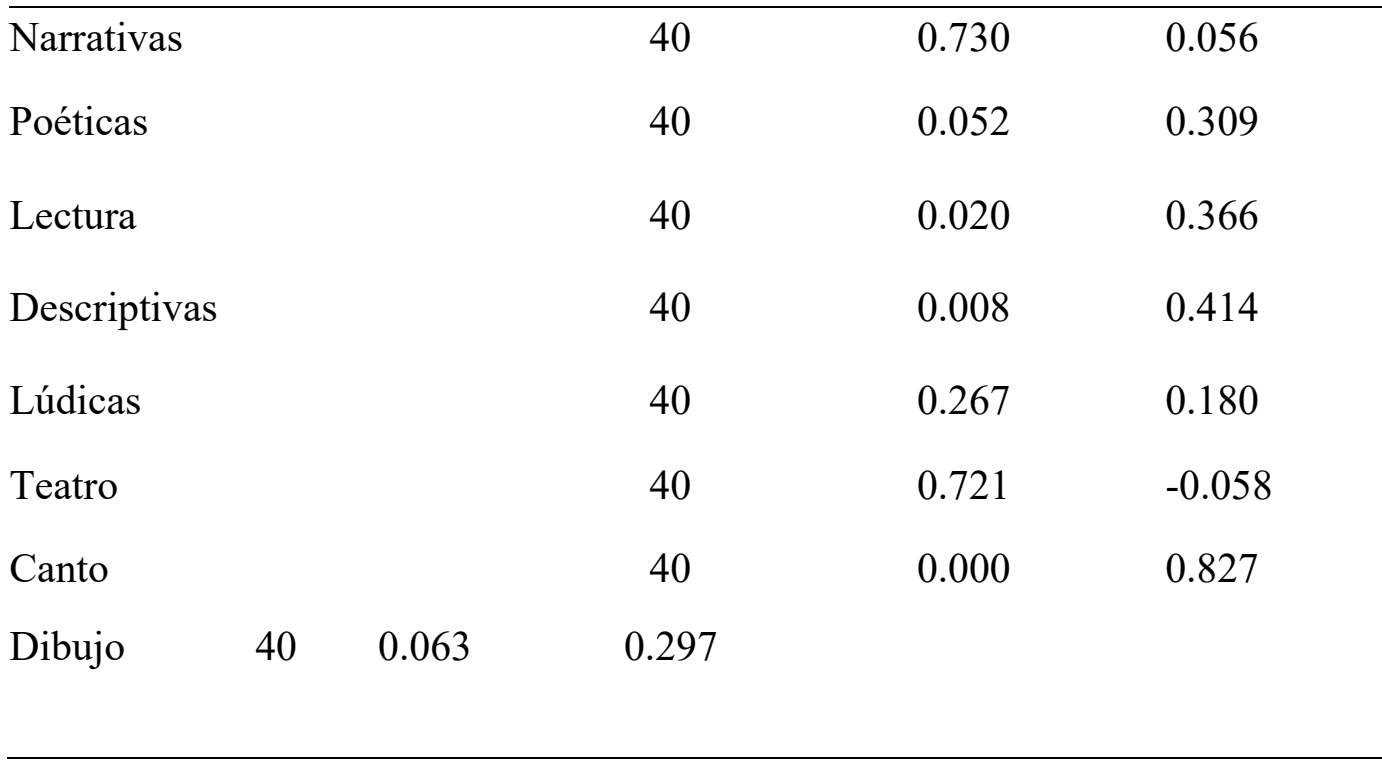

En el resumen del análisis de correlación de las diferentes estrategias didácticas y la comunicación familiar, se describen los resultados de la relación: las estrategias narrativas $\left(r_{s}=0.056, p=0.730\right)$, estrategias poéticas $\left(r_{s}=0.309, p=0.052\right)$, estrategias lúdicas $\left(r_{s}=0.180, p=0.267\right)$, estrategias de teatro $\left(r_{s}=-0.058, p=0.721\right)$ y estrategias de dibujo $\left(r_{s}=0.297, p=0.063\right)$; la relación estadísticamente no es significativa con la dimensión comunicación familiar a un $\alpha=5 \%$. Las estrategias didácticas que mostraron relación con la dimensión comunicación familiar son: las estrategias didácticas de lectura presentan una correlación baja y significativa $\left(\mathrm{r}_{\mathrm{s}}=0.366, \mathrm{p}=0.020\right)$, del mismo modo las estrategias didácticas descriptivas presentan una correlación moderada y significativa $\left(\mathrm{r}_{\mathrm{s}}=0.414\right.$, $\mathrm{p}=0.008)$ y una relación muy alta y significativa $\left(\mathrm{r}_{\mathrm{s}}=0.827, \mathrm{p}=0.000\right)$ presentó la estrategia didáctica del canto.

Relación entre estrategias didácticas y comunicación escolar Tabla 6

Relación entre las dimensiones de las estrategias didácticas y comunicación escolar

$$
\text { Comunicación escolar }
$$

Estrategias didácticas

\begin{tabular}{lll}
\hline $\mathrm{n}$ & $\mathrm{p}$
\end{tabular}

Narrativas

$40 \quad 0.420$

0.131

Poéticas

$40 \quad 0.015$

0.383 
Lectura

Descriptivas

Lúdicas

Teatro

Canto

Dibujo
$40 \quad 0.018$

$40 \quad 0.019$

$40 \quad 0.473$

$40 \quad 0.774$

40 .

0.370
0.373

0.369

0.117

$-0.047$

1.000

El análisis de relación entre las diferentes estrategias didácticas y la dimensión comunicación escolar de la tabla 03, evidencia que no existe relación estadísticamente significativa entre las estrategias narrativas $\left(\mathrm{r}_{\mathrm{s}}=0.131, \mathrm{p}=0.420\right)$, estrategias lúdicas $\left(\mathrm{r}_{\mathrm{s}}=0\right.$., $\mathrm{p}=0.052)$, y estrategias de teatro $\left(\mathrm{r}_{\mathrm{s}}=0 .-0.047, \mathrm{p}=0.774\right)$; sin embargo se encontró evidencias estadísticamente significativas para las demás estrategias: relación baja y significativa para las estrategias didácticas poética $\left(\mathrm{r}_{\mathrm{s}}=0.383, \mathrm{p}=0.015\right)$, de lectura $\left(\mathrm{r}_{\mathrm{s}}=0.373, \mathrm{p}=0.018\right)$, descriptivas $\left(\mathrm{r}_{\mathrm{s}}=0.369, \mathrm{p}=0.019\right)$ y de dibujo $\left(\mathrm{r}_{\mathrm{s}}=0.370, \mathrm{p}=0.019\right) ;$ la estrategia didáctica $\left(\mathrm{r}_{\mathrm{s}}=1\right)$, una aparente relación perfecta. Todos los resultados fueron evaluados con una significancia $\alpha=5 \%$.

\section{DISCUSIÓN}

En el estudio participaron 40 niños de cinco años de edad, quienes proporcionaron información para el cuestionario de estrategias didácticas y comunicación; los datos obtenidos por el cuestionario fueron ingresados al IBM SPSS Statistics V22 previa declaración de variables consideradas en el estudio, considerando el criterio de eliminación de casos con el 10\% a más de ítems perdidos por variables, se redujo el número de los participantes a 40 individuos; esta eliminación corresponde especialmente a aquellos niños, cuyo indicador evaluado no estuvo presente. Se realizó el análisis correspondientes para las puntuaciones finales obtenidas en cada una de las variables, evaluando los supuestos que exigen las pruebas paramétricas; la prueba de normalidad (Shapiro-Wilk=0.946, $\mathrm{p}=0.054$ ) indica una aproximación a una distribución normal de las puntuaciones para la variable: estrategias de comunicación y para la variable comunicación (Shapiro-Wilk=0.279, $\mathrm{p}=0.000$ ); indica que las puntuaciones de la variable comunicación no tienen una aproximación a una distribución normal; por lo tanto, al no 
cumplir con este supuesto se procedió a aplicar la prueba no paramétrica de correlación de Spearman, sobre la base de las puntuaciones totales y por dimensiones de las variable según el diseño planteado de la investigación. Según los resultados obtenidos, la aplicación de las estrategias didácticas favorece la comunicación, en los niños y niñas del nivel inicial. La estrategia más "eficiente" es mediante el canto.

Por su parte, Rodríguez Silva (2013) ha investigado con el objetivo de "proponer nuevos métodos, técnicas, estrategias de enseñanza aplicando a la música"; ha subrayado los beneficios de la música para los niños, en quienes desarrolla sus capacidades y destrezas durante los primeros años de educación, cuya investigación fue desarrollada en el contexto de un estudio básico, bibliográfico-documental, exploratorio, en 66 niños de ambos géneros. Pérez-Aldeguer (2014) destaca la importancia del canto coral, para beneficio de los niños, cuyos han corrobora tal importancia desde los puntos de vista de "la medicina, la etnomusicología o la psicología". Mónaco (2012) destaca la importancia de la música, inclusive antes de la escuela, en el vientre de la madre, refiere la edad de cuarenta y cinco meses de gesta. Los niños de 3 y años tienen la mayor posibilidad de ingresar en su entorno, gracias a la música.

Además, la docente de aula busca las estrategias más relevantes, con el propósito de aplicarlas en su trabajo pedagógico, de acuerdo con las necesidades y las condiciones de sus alumnos. La aplicación de las estrategias didácticas, en las aulas del nivel de inicial, ha permitido que nuestros alumnos y alumnas sean más participativos, más receptivos a los nuevos aprendizajes; quienes tienen la iniciativa de realizar sus propias creaciones y de exponerlas. La música y el canto mejoran el desarrollo integral de los niños.

\section{CONCLUSIONES}

Las estrategias didácticas tienen una relación significativa con la comunicación en los niños y niñas de 5 años de la villa Monte Castillo: Catacaos, Piura, Perú, 2014. El uso adecuado de las estrategias didácticas (narrativa, poética, lectura, descriptiva, lúdicas, teatro, canto y dibujo) tiene una relación significativa con la comunicación familiar de los niños. Es decir, estas estrategias permiten que los niños y niñas desarrollen su comunicación familiar. El uso adecuado de estas estrategias didácticas tiene una relación significativa con la comunicación escolar de los niños. Es decir, permiten que los niños y niñas desarrollen su comunicación escolar. Por otro lado, las estrategias didácticas permiten que el aprendizaje sea más agradable y más receptivo para nuestros estudiantes, 
permitiendo así incrementar su vocabulario, ser más sociables, más participativos en las diferentes actividades propuestas por la docente.

Asimismo, las diferentes actividades propuestas, durante las actividades pedagógicas, ayudan a los educandos a meditar en cómo y cuánto han aprendido, cómo ha sido su comportamiento durante las sesiones de clase, reconociendo sus dificultades y logros obtenidos. Los docentes deben bridar un abanico de estrategias didácticas en el área de comunicación, para que los niños y niñas desarrollen su creatividad, sus habilidades, destrezas, su imaginación, la práctica de valores y aprendan a buscar soluciones.

\section{REFERENCIAS}

Alvites, G. (2012). Estilos educativos y grados de desarrollo del componente pragmático del lenguaje en niños de 5 años de un colegio de Lima Metropolitana. [Tesis para el grado de magíster, Pontificia Universidad

$$
\text { Católica del Perú]. Retrieved from }
$$

http://tesis.pucp.edu.pe/repositorio/bitstream/handle/20.500.12404/1646/

\section{ALVITES_SOSA_GONY_ESTILOS_EDUCATIVOS.PDF? sequence $=1$}

Campo, L. (2010). Importancia del desarrollo motor en relación con los procesos evolutivos del lenguaje y la cognición en niños de 3 a 7 años de la ciudad de Barranquilla (Colombia). Salud Uninorte, 26(1), 65-76. Retrieved from https://www.redalyc.org/pdf/817/81715089008.pdf

Garcés, M., and Palacio, J. (2010). La comunicación familiar en asentamientos subnormales de montería (Colombia). Psicología Desde
El Caribe,
1-29. Retrieved
from

http://rcientificas.uninorte.edu.co/index.php/psicologia/article/viewFile/3 7/604

Guaita, V. L. (2010). La evaluación de la percepción de la comunicación emocional de los padres en niños desde la neuropsicología.

Interdisciplinaria, 27(1), 129-146. Retrieved from https://www.redalyc.org/pdf/180/18014748009.pdf Hernández Sampieri, R., Fernández Collado, C., and Baptista Lucio, M. del P. (2014).

Metodología de la Investigación (Sexta edic). México, D.F:

McGraw-Hill Interamericana Editores, S.A. de C.V.

Melquiades, A. (2014). Estrategias didácticas para un aprendizaje constructivista en la enseñanza de las matemáticas en los niños y niñas de nivel primaria. Textos Y Contextos, 43-58. Retrieved from file:///E:/Estrategias didácticas para niños de 5 
años/DialnetEstrategiasDidacticasParaUnAprendizajeConstructivi6349169.pdf

Mendoza, S., and Pabón, J. (2013). Propuesta didáctica para el desarrollo del pensamiento lógico-matemático en niños de 5 años. [Tesis de posgrado,

Universidad Pedagógica Nacional]. Retrieved from

http://repositorio.pedagogica.edu.co/bitstream/handle/20.500.12209/506/

TO16309.pdf? sequence $=1$ \&isAllowed $=\mathrm{y}$

Mónaco, M. (2012). Cantar canciones entre los cuarenta y cincuenta meses: una habilidad en gestación. [Tesis presentada para obtener el grado de Master en Psicología de la Música, Universidad Nacional de La Plata].

Retrieved from

http://sedici.unlp.edu.ar/bitstream/handle/10915/26425/Documento_com

pleto__.pdf? sequence $=1 \&$ isAllowed $=\mathrm{y}$

Montenegro, M., and Valencia, J. (2013). Aplicación del material didáctico para el desarrollo de lenguaje escrito en niños de 4-5 años de los centros de educación inicial "Piedad Gómezjurado”, "Francisco Javier Salazar” y "Rincón del Saber." [Tesis de licenciatura, Universidad

$\begin{array}{llll}\text { Técnica } & \text { del } & \text { Norte]. } & \text { Retrieved }\end{array}$ http://repositorio.utn.edu.ec/bitstream/123456789/2546/1/05 FECYT 1825.pdf

Orellana-García, P., and Melo-Hurtado, C. (2014). Ambiente letrado y estrategias didácticas en la educación preescolar chilena. Magis, Revista Internacional de Investigación En Educación, 6(13), 113-128. https://doi.org/10.11144/Javeriana.M613.ALED

Paredes, N. (2011). La estimulación temprana del hogar en el desarrollo del lenguaje de los niños y niñas de 4 a 5 años del centro educativo particular "Mi Nuevo Mundo" dela ciudad de Quito. Año lectivo 20102011. [Tesis de licenciatura, Universidad Central del Ecuador]. Retrieved from http://www.dspace.uce.edu.ec:8080/bitstream/25000/304/1/TUCE001071.pdf

Pérez-Aldeguer, S. (2014). El canto coral: una mirada interdisciplinar desde la educación musical. Estudios Pedagogicos, XL(1), 389-404. Retrieved from https://scielo.conicyt.cl/pdf/estped/v40n1/art23.pdf

Rodríguez Silva, A. E. (2013). Los beneficios de la música incide en el desarrollo de las capacidades y destrezas de los niños(as) de los primeros años de educación general básica de la Escuela Fiscomisional, La Merced, de la Parroquia Izamba, Cantón Ambato. 
[Tesis para la obtención del título, Universidad Técnica de Ambato]. Retrieved from https://repositorio.uta.edu.ec/bitstream/123456789/6903/1/FCHECEP537.pdf

Telpis, M., and Terán, S. (2014). La literatura infantil como instrumento idóneo para el desarrollo del pensamiento creativo y sensibilidad literaria en la educación inicial para los niños y niñas de 4 a 5 años, en el centro de educación inicial "Rafael Suárez", de la ciudad de Ibarra, en. [Tesis de licenciatura, Universidad Técnica del Norte]. Retrieved from file://E:/Estrategias didácticas para niños de 5 años/05 FECYT 2060 TESIS.PDF Trujillo, M. (2013). Desarrollo psicomotor y las capacidades del área de comunicación en los niños de 5 años del nivel inicial de la I. E. "Santa Rosa" $N^{o}$ 20501, PativilcaBarranca, 2013. [Tesis de magíster,

Universidad César Vallejo]. Retrieved from http://repositorio.ucv.edu.pe/bitstream/handle/20.500.12692/13892/Trujil 1o_SMA.pdf?sequence $=1$

Valdés, M., and Spencer, R. (2011). Influencia del nivel socioeconómico familiar sobre el desarrollo psicomotor de niños y niñas de 4 a 5 años de edad de la ciudad de Talca Chile. Theoria, 20(2), 29-43. Retrieved from http://revistas.ubiobio.cl/index.php/RT/article/view/1249 